Elsevier

HRR 00947

\title{
Effects of outer hair cell loss on the frequency selectivity of the patas monkey auditory system
}

\author{
D.W. Smith *, D.B. Moody, W.C. Stebbins, and M.A. Norat \\ Kresge Hearing Research Institute, University of Michigan Medical School, Ann Arbor, U.S.A. and Dept. of Psychology, \\ University of Michigan, Ann Arbor, U.S.A.
}

(Received 27 August 1986; accepted 25 March 1987)

\begin{abstract}
This report describes a study that took advantage of the unique reactivity of the patas monkey (Erythrocebus patas) to dihydrostreptomycin-sulfate (DHSM) to investigate the effects of selective outer hair cell (OHC) lesions on psychophysical tuning curves (PTC). Four patas monkeys were trained using operant reinforcement techniques to perform forward masking PTCs at frequencies of $500 \mathrm{~Hz}, 2,4$, and $8 \mathrm{kHz}$, at $10 \mathrm{~dB} \mathrm{SL}$. Steady and pulsed-tone thresholds were also measured from $63 \mathrm{~Hz}$ to $40 \mathrm{kHz}$ in half-octave steps. The animals were given daily i.m. injections of DHSM at $20 \mathrm{mg} / \mathrm{kg}$ per day until shifts in absolute threshold at 16 $\mathrm{kHz}$ exceeded $10 \mathrm{~dB}$, at which time the drug was discontinued. Initial changes in PTC shape included elevations in the tip region associated with the increase in threshold and no elevation or a hypersensitivity of the low-frequency tail region. In general, threshold and therefore PTC tip elevations of at least $40 \mathrm{~dB}$ were required before any increase in the low-frequency tail became evident. Following completion of psychophysical testing, animals were sacrificed and cytochochleograms were determined. At frequencies corresponding to regions of complete OHC loss and complete IHC retention a lack of selectivity was evident and PTCs closely resemble low-pass filters. This residual low-pass tuning is similar to that seen in VIIIth nerve fibers in ears devoid of OHCs and in basilar membrane transfer functions from traumatized ears. PTCs taken at frequencies corresponding to areas with no loss of receptors showed no systematic changes in sensitivity or selectivity. Because loss of normal OHC function results in greater than a 50-dB loss in sensitivity, as well as a detuned PTC, these findings strongly support the suggestion that the role of the OHC system is to increase the sensitivity and selectivity of the auditory system.
\end{abstract}

Frequency selectivity; Psychophysical tuning curve; Hair cell, outer

\section{Introduction}

Frequency selectivity, the process by which the ear resolves the individual components of an acoustic complex, represents one of the most critical functions of the auditory system, mediating both the perception of speech and the binaural localization of sound in space. Traditional models of cochlear function have emphasized the passive mechanical properties of the basilar membrane in determining the frequency selectivity and sensitivity of the auditory system (Békésy, 1960). In this

Correspondence to: D.B. Moody, Ph.D. Kresge Hearing Research Institute, University of Michigan Medical School, Ann Arbor, MI 48109, U.S.A.

* Present address: Human Communications Laboratories, Boys Town National Institute, 555 N. 30th Street, Omaha, NE 68131, U.S.A. scheme, the hair cells are passive receptors of mechanical stimulation from the sharply tuned cochlear partition. However, recent descriptions of the distribution of contractile proteins by Flock (1983), as well as the discovery of active properties of the outer hair cell (OHC) system by Brownell (1983) and Zenner et al. (1985), have forced radical revisions of these views.

Evans (1973, 1975), Evans and Wilson (1973, 1975), and, more recently, Khanna (1984) have suggested that the frequency selectivity of the auditory neuron and basilar membrane consist of two components. The first is a low-pass filter process, apparently dependent upon the passive mechanical properties of the basilar membrane and surrounding fluids, and is relatively immune to trauma. The second component is a sharply tuned response near the high-frequency cut-off 
point of the low-pass system. This second component, quite unlike the first, is physiologically labile.

Numerous lines of evidence and most current models suggest that the active mechanical properties of the $\mathrm{OHC}$ subsystem are responsible for the sharply tuned response of the system as a whole. For example, Khanna and Leonard (1986) and Leonard and Khanna (1984) have shown that both the sensitivity and tuning of the basilar membrane, as measured by laser interferometry, are related to the condition of the OHC system. Likewise, Liberman and Dodds (1984) have shown that the sharpness of 8 th nerve single-unit tuning curves (FTCs) arising from identified inner hair cells (IHCs) is a function of the condition of the adjacent $\mathrm{OHCs}$, specifically the condition of their ciliary tufts.

While studies of these phenomena in isolation have shed a lot of light on the physiological basis of the auditory system and hearing per se, they tell us little about the role of these processes in behavior. However, that issue may be addressed directly by questioning the intact organism within a behavioral paradigm. Systematic changes in psychophysical measures following controlled lesioning of various structures provide a direct description of their function in hearing.

In the only report directly addressing the issue in the extant literature, Dallos et al. (1977) looked at the effects of selective OHC loss on both 8th nerve FTCs and psychophysical tuning curves (PTCs) in chinchillas. Following kanamycin treatment, which produces a selective OHC loss, FTCs taken at frequencies corresponding to regions of $\mathrm{OHC}$ damage were elevated and detuned. However, PTCs taken from areas of the organ of Corti devoid of $\mathrm{OHCs}$ exhibited increased thresholds, yet, unlike the physiological tuning curves, retained sharp tuning. These data, according to Dallos et al. (1977), indicate an increased tuning above the level of the auditory nerve. However, they are not in agreement with the extensive human literature that indicates that auditory bandwidth increases and speech perception abilities deteriorate with hearing losses of cochlear origin (c.f., Zwicker, 1974, 1983; Pick et al., 1977; Wightman et al., 1977; Wightman, 1982) and therefore suggest a decrease in the frequency resolving power of the system as a whole.
The reasons for the discrepancies between the behavioral and physiological findings are not clear and further systematic analyses which directly relate changes in structure to alterations in psychophysical frequency selectivity are necessary. We sought to examine the function of the $\mathrm{OHC}$ system in determining frequency selectivity by taking advantage of the unique reactivity of the patas monkey to dihydrostreptomycin-sulfate (DHSM). Systemic treatment with DHSM in the patas monkey produces a selective loss of OHCs, while leaving IHCs intact. Since the PTC represents a critical descriptor of normal auditory processing, changes in this index during selective loss of OHCs can tell us much about their role in normal cochlear function.

\section{Materials and Methods}

\section{Subjects}

The subjects in these studies were four juvenile male patas monkeys (Erythrocebus patas). The animals were maintained on a restricted diet to facilitate behavioral training and testing procedures. Their weights ranged from approximately 4 to $8 \mathrm{~kg}$ during the 4.5 year course of these studies. The subjects had normal hearing at all frequencies prior to drug treatment as determined by steady and pulsed-tone behavioral threshold procedures.

\section{Apparatus}

During testing procedures the subjects were seated in primate restraint chairs with their heads immobilized. Headphones were fitted as closely as possible over the pinna, and a feeder trough was mounted on the chair within easy reach of the subject's mouth. A response manipulandum was placed in front of the chair and contained a cue light to signal the experimental conditions to the subjects at all times. All test sessions were conducted in double-walled sound attenuating chambers. Stimulus presentation and experimental parameters were under computer control.

\section{Stimuli}

The stimuli in these studies were pure-tones generated by Hewlett-Packard oscillators (models $204 \mathrm{C}$ and $200 \mathrm{CDR}$ ) which were fed through a computer controlled relay, tone switch and at- 
tenuation network to TDH-49 headphones. The headphones (with MX-41/AR cushions) were calibrated using a Brüel and Kjaer $1 / 2$ inch condenser microphone (type 4134) with a 6 cc coupler and a Hewlett-Packard wave analyzer (model 3590A). All stimuli had raised-cosine envelopes and durations were measured at the 3-dB down points.

\section{Behavioral procedure}

Pure tone thresholds were measured with hoth steady and pulsed tones at frequencies from $63 \mathrm{~Hz}$ to $40 \mathrm{kHz}$. Thresholds were measured to $2.5-\mathrm{s}$ steady tones (5-ms rise/fall) to determine the functional status of the experimental ear prior to drug treatment. Thresholds to $25-\mathrm{ms}$ pulsed tones (5-ms rise/fall) were measured daily to allow setting of the PTC probe tone level relative to threshold.

Forward-masking psychophysical tuning curves were measured at frequencies of $500 \mathrm{~Hz}, 2,4$, and $8 \mathrm{kHz}$. All measurements were taken with the probe stimulus level at $10 \mathrm{~dB}$ SL. The maskers and probe tones had durations of 130 and $25 \mathrm{~ms}$, respectively. Both the masker and probe tones were pure tones gated with random phase and had rise/fall times of $5 \mathrm{~ms}$. There was a 10-ms delay between $50 \%$ masker offset and $50 \%$ probe tone onset and a 130-ms delay between masker offset and onset of the subsequent masker.

The subjects were trained using positive reinforcement procedures that have been described in greater detail elsewhere (Moody et al., 1975; Serafin et al., 1982). The beginning of the trial sequence was signalled to the subject by the flashing cue light. The subject was then required to make contact with the manipulandum. When contact was made, the cue light then remained on without flashing for the duration of the trial. Following a variable hold of 1-9 s, with an average delay of 5 $\mathrm{s}$, a test interval was presented. The subject was required to release the lever during the test interval. All correct releases of the lever during the test-signal presentations were followed by delivery of a banana-flavored food pellet reinforcer (190 mg, Bioserv) to the feeder trough.

Releases of the lever at any other time during the trial sequence resulted in a 5-s time-out period during which the cue light was extinguished and the subject was required to wait before initiating another trial sequence. The time out was mildly aversive and served to decrease inappropriate responding. The test interval for the steady-tone threshold procedure involved the presentation of a single 2.5-s tone. For the pulsed tone, the interval consisted of the presentation of $1025-\mathrm{ms}$ pulses, each separated by $235 \mathrm{~ms}$, resulting in a total test duration of $2.6 \mathrm{~s}$. The level of the test tone from trial to trial was varied using a 'staircase' or tracking procedure. The initial level of the stimulus was set approximately $50 \mathrm{~dB}$ above threshold and each correct detection resulted in a $10-\mathrm{dB}$ decrease in test-tone level on the subsequent trial. This procedure continued until the subject failed to report the presence of the tone, at which point its level was increased $10 \mathrm{~dB}$ for the next presentation. This change, from a detection to a miss, or vice versa, defined a 'transition'. Seven transitions were measured at each frequency and thresholds were defined as the average SPL over the last 5 transitions. Thresholds at a given frequency were measured twice each session and the values averaged to specify that session's threshold value.

Baseline data collection for both steady and pulsed-tone thresholds continued until threshold values stabilized and 8 of 10 consecutive session thresholds were within $10 \mathrm{~dB}$ at a given frequency. The average of these 8 thresholds was taken as the baseline value.

The trial sequence for PTCs varied slightly from that for absolute thresholds in that initial contact with the manipulandum produced a train of $130-\mathrm{ms}$ pure-tone masking pulses. Following a variable number of maskers, the test interval was presented where $25-\mathrm{ms}$ probe tones (i.e., the same pulses used in the absolute threshold procedure) followed each of 10 consecutive maskers.

Like the threshold testing procedures, the level of the masker from trial to trial was set using a tracking procedure. The initial level of the masker was set at a value considerably below levels required to mask the test tone. Correct detection of the test signal by the subject produced a $10-\mathrm{dB}$ increase in the level of the masker for the subsequent trial. This procedure continued until the subject failed to detect the presence of the probe tone, whereupon the masker level was decreased $10 \mathrm{~dB}$ for the next trial presentation. Again, the 
change from a detection to a miss, or vice versa, defined a transition. Seven transitions were measured at each masker frequency and a masker threshold was defined as the average SPL over the last 5 transitions. Masker thresholds at each frequency were measured twice during each session and averaged to produce the session's threshold value. A minimum of 9 , and up to 18 masker frequencies were tested for each PTC.

The stability criterion for baseline PTC measurements was 5 consecutive sessions with masker threshold at the PTC frequency within $5 \mathrm{~dB}$. Baseline PTCs were defined as the average of the masker values at each frequency from these 5 sessions.

In order to assess the subject's guess rate, $20 \%$ of all test tone periods were 'catch trials' where all trial parameters were identical to test-tone trials except the probe tone was withheld. Responses during catch trials resulted in a 5-s time out. Data from sessions with a greater than $20 \%$ response to catch trials were discarded and not used in summary data calculations.

Post-drug PTC and threshold measurements continued until no systematic changes were evident in the data for 30 days, at which point summary data collection, using the baseline stability criteria, was concluded.

\section{Drug administration}

Following collection of baseline data each subject underwent treatment with DHSM. Daily intramuscular injections of DHSM were given at a dose of $20 \mathrm{mg} / \mathrm{kg}$ per day. Like other ototoxic aminoglycoside antibiotics, DHSM produces a progressive loss of hearing beginning at the high frequencies. In the DHSM/patas monkey model, this loss invariably begins between the 6th and 9 th weeks of drug treatment. Daily injections were discontinued when a permanent threshold shift of $10 \mathrm{~dB}$ was evident at $16 \mathrm{kHz}$. However, unlike other aminoglycosides, the hearing loss from DHSM continues following drug cessation, sometimes for up to 6 months.

\section{Histological preparation}

Following completion of post-drug summary data collection, the animals were euthanized and temporal bones removed for histological evalua- tion. The procedures used in preparing the ear for examination were carried out using standardized techniques. Briefly, initial fixation of the ear was done by in vivo perilymphatic perfusion of Karnovsky fixative (4\% paraformaldehyde, 5\% glutaraldehyde) through the round and oval windows following a radical mastoidectomy and removal of the ossicular chain. The chest was then opened and the animal given a cardiac perfusion

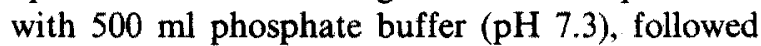
by 11 half-strength Karnovsky fixative (2\% paraformaldehyde, $2.5 \%$ glutaraldehyde). The ears were then removed, reperfused, and placed in a Karnovsky bath overnight. The ear then went through a series of buffer washes followed by a post-fix in osmium tetroxide $(1 \%)$. The bone was then dehydrated in a series of graded ethyl alcohol baths, dissected, and tissues embedded in Medcast-araldite molds. Cytocochleograms were obtained from each ear under light microscopy. Post-drug IHC condition was evaluated by taking cross-sections of the flat-molds in $5-\mu \mathrm{m}$ sections from regions where there was a loss of IHCs and retention of OHCs.

\section{Results}

While the extent of the hearing loss and range of affected frequencies varied from subject to subject, the systematic changes seen in PTCs measured from regions of OHC loss are in agreement for all subjects and frequencies where a shift in thresholds was evident. For this reason the changes in sensitivity and tuning will be illustrated by examination of the individual data from two subjects, M-155 and M-157. These two subjects were chosen because the alterations in frequency selectivity accompanying $\mathrm{OHC}$ loss were very slow and orderly and therefore best documented. In one of the two remaining subjects, threshold shifts occurred so rapidly that the day-to-day systematic changes could only be followed at $8 \mathrm{kHz}$. The remaining subject, whose systematic changes in frequency selectivity were identical to the other animals, required longer drug treatments to produce initial shifts in thresholds and subsequently showed a terminal $40-60 \%$ loss of IHCs throughout the ear. This initial resistance to DHSM, and subsequent significant loss of IHCs 


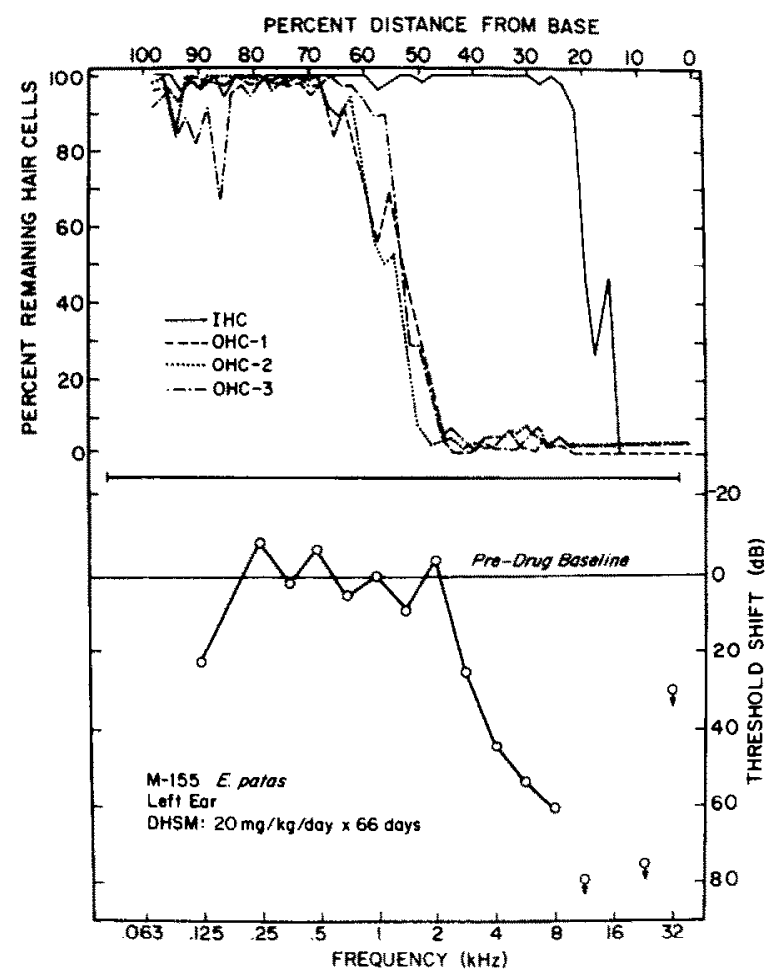

Fig. 1. Cytocochleogram (upper panel) for M-155, plotted as percent remaining hair cells as a function of distance along the basilar membrane from base. Corresponding final audiogram (lower panel) for M-155 is given as steady tone threshold shift as a function of frequency. Actual length of basilar membrane, $22.59 \mathrm{~mm}$

was unique to the subject and might unnecessarily complicate the analysis of the data.

Changes in PTC shape will be described in qualitative rather than quantitative terms for two reasons: first, mathematical measures of filter shape such as $Q_{10}$ describe symmetrical filter functions. However, changes in PTCs in response to pathology, like changes in FTCs, are highly asymmetric, and therefore these measures are rendered ineffective. Second, PTCs lose bandpass function rapidly with loss of OHCs which precludes use of the typical quantitative measures of filter shape.

A potential confounding factor in using the PTC to analyze functional changes in pathological cochleae is the finding that PTC shape is dependent on the absolute level at which measurements are taken (Nelson, 1980; Green et al., 1981; Zwicker, 1974; Nelson and Freyman, 1984). At

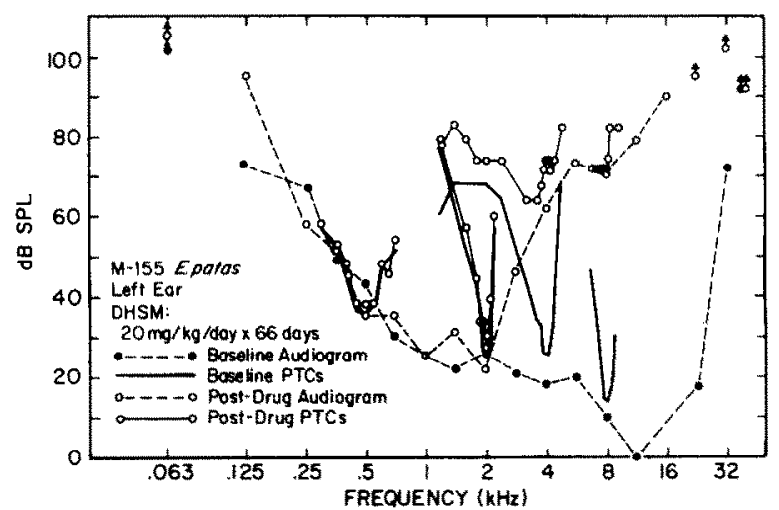

Fig. 2. Direct comparison of baseline and post-drug pulsed-tone threshold curves and PTCs for M-155.

higher stimulus levels it becomes necessary to differentiate between changes brought on by pathology and those by higher stimulus levels, per se. Tuning curves broaden as the absolute level of the signal is increased. Since a loss of OHCs is associated with a 40-60 dB increase in threshold, postdrug measurement levels will necessarily be higher than those prior to drug administration. To serve as comparison, therefore, tuning curves obtained at 30 and $60 \mathrm{~dB}$ SL probe tone levels from the same subjects prior to drug treatment are presented (Smith et al., 1987). These levels represent the highest absolute SPLs which might be encountered with a severe hearing loss. The tuning curves were gathered using the same equipment and protocol described above. Comparisons of pathological PTCs with abnormally elevated thresholds with normal PTCs taken at roughly equivalent SPLs allows the differentiation of changes which are a function of pathology from those which are a function only of testing at increased sound levels.

The apper panel of Fig. 1 presents the cytocochleogram for subject $\mathrm{M}-155$, plotted as percent remaining hair cells as a function of distance along the basilar membrane from the base. The lower panel presents the corresponding audiogram showing steady-tone threshold shift as a function of frequency. The basilar membrane distance and frequency coordinates have been arbitrarily aligned such that the midpoint of the membrane corresponds to a frequency of $1.6 \mathrm{kHz}$.

The hair cell count for M-155 showed an absence of IHCs in the basal $15 \%$ of the cochlea with a rapid transition to nearly complete retention of 


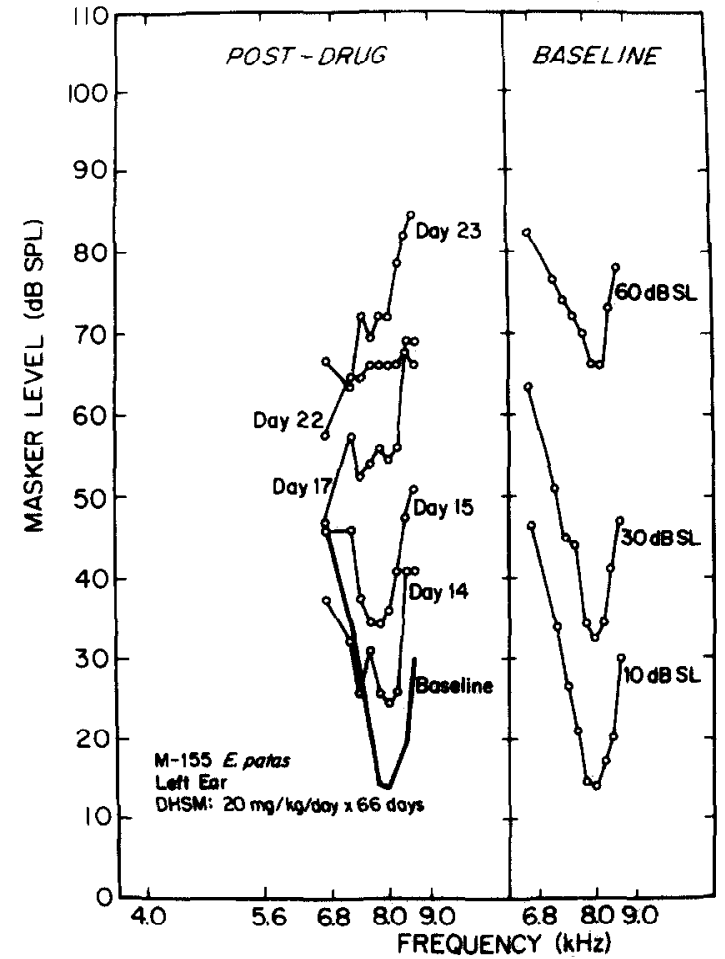

Fig. 3. Systematic progression of changes in $8 \mathrm{kHz}$ PTC for M-155 measured on various days post-drug (left panel). Normal PTCs measured at high SPLs from Smith et al. (1987) are presented for comparison at 10,30 and $60 \mathrm{~dB}$ (right panel).

IHCs throughout the apical $80 \%$. Light microscopic evaluation of IHC condition in these regions revealed normal-looking cells in which stereocilia were present and of normal shape and configuration, the cell body had normal shape and size, and there was no evidence of vacuolization within the cell. The OHCs were absent from the basal half with a transition to nearly normal populations in the apical half of the cochlea. This pattern of loss produced a region with normal IHCs and devoid of OHCs over approximately one-third of the basilar membrane.

The frequencies of the accompanying threshold shifts correspond closely to the regions of receptor damage. A loss of 22 to $60 \mathrm{~dB}$ was evident at frequencies between 2.8 and $8 \mathrm{kHz}$, corresponding to the regions of selective IHC retention. The threshold shifts were so large as to preclude measurement with the available equipment at frequencies above $8 \mathrm{kHz}$. Thresholds were normal at 2 $\mathrm{kHz}$ and below, except for a $20 \mathrm{~dB}$ shift at $125 \mathrm{~Hz}$.
Fig. 2 presents a direct comparison of baseline and post-drug pulsed-stimulus thresholds and PTC plots for $\mathrm{M}-155$. At $8 \mathrm{kHz}$, corresponding to a region of complete $\mathrm{OHC}$ loss and normal $\mathrm{IHC}$ populations, the threshold shift was $60 \mathrm{~dB}$ and the resulting PTC resembled a low-pass filter. At 4 $\mathrm{kHz}$ the PTC was elevated approximately $40 \mathrm{~dB}$, yet still exhibited some tuning since the low frequency tail overlaps the $2 \mathrm{kHz}$ region of normal post-drug sensitivity and selectivity function. The high frequency tails of both 4 and $8 \mathrm{kHz}$ PTCs were approximately $10 \mathrm{~dB}$ above the pulsedstimulus threshold curve. The post-drug PTCs at $500 \mathrm{~Hz}$ and $2 \mathrm{kHz}$, where thresholds to both steady and pulsed stimuli were unaffected, overlie the baseline functions.

The left panel of Fig. 3 shows the progressive changes in tuning evident for M-155 during loss of threshold sensitivity at $8 \mathrm{kHz}$ measured on various days post-drug. The right panel shows normal PTCs for M-155 measured at probe-tone levels of 10, 30, and $60 \mathrm{~dB}$ SL from Smith et al. (1987) for comparison. The initial changes in tuning curve shape were consistently seen as two distinct phenomena. The first was observed as an elevation and shift of the characteristic frequency (CF) toward lower frequencies. Concomitant with elevations of up to $50 \mathrm{~dB}$ was either no change, or a hypersensitivity in the low-frequency tail. At days 14,15 , and 17 the low-frequency tail at $6.8 \mathrm{kHz}$ was either equal to, or less than baseline values, while a greater than $40-\mathrm{dB}$ loss in tip sensitivity occurred within those 3 days. By days 22-23 the function was elevated nearly $60 \mathrm{~dB}$ and resembled a low-pass filter process.

A comparison of post-drug filter functions with normal PTCs taken at roughly equivalent SPLs (right panel) suggests the lack of tuning in pathological PTCs cannot be accounted for on the basis of increases in measurement level. Changes caused by increases in SPL were a decrease in tip-to-tail ratio, and a slight broadening of the filter function. The changes with loss of $\mathrm{OHCs}$ were a complete detuning and transition from bandpass to low-pass function.

Fig. 4 presents the progressive changes in 4 $\mathrm{kHz}$ PTCs for M-155. An average of the data from days 36-39 showed a 30-dB elevation and shift toward lower frequencies at the $\mathrm{CF}$, while a hyper- 


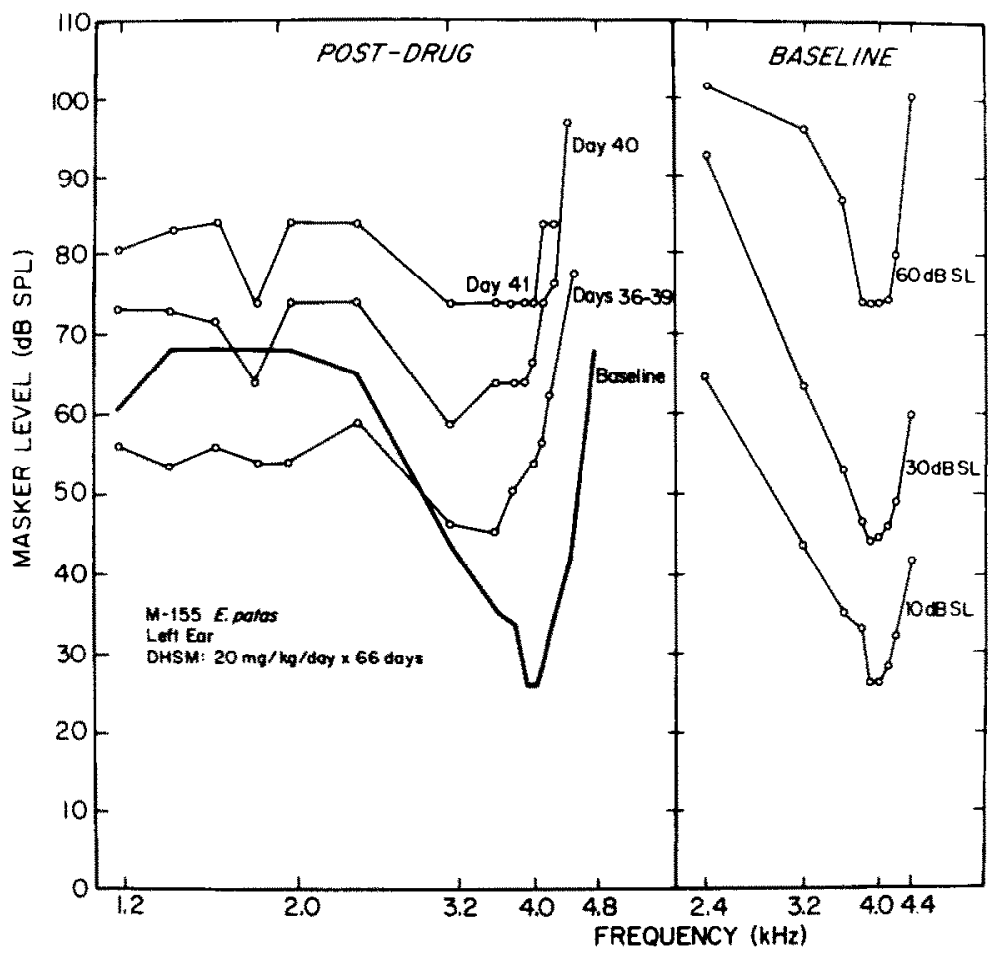

Fig. 4. Systematic changes in $4 \mathrm{kHz}$ PTC for M-155 measured on various days post-drug (left panel). Normal PTCs for same subject at 10,30, and $60 \mathrm{~dB}$ SL are presented for comparison (right panel), from Smith et al. (1987).

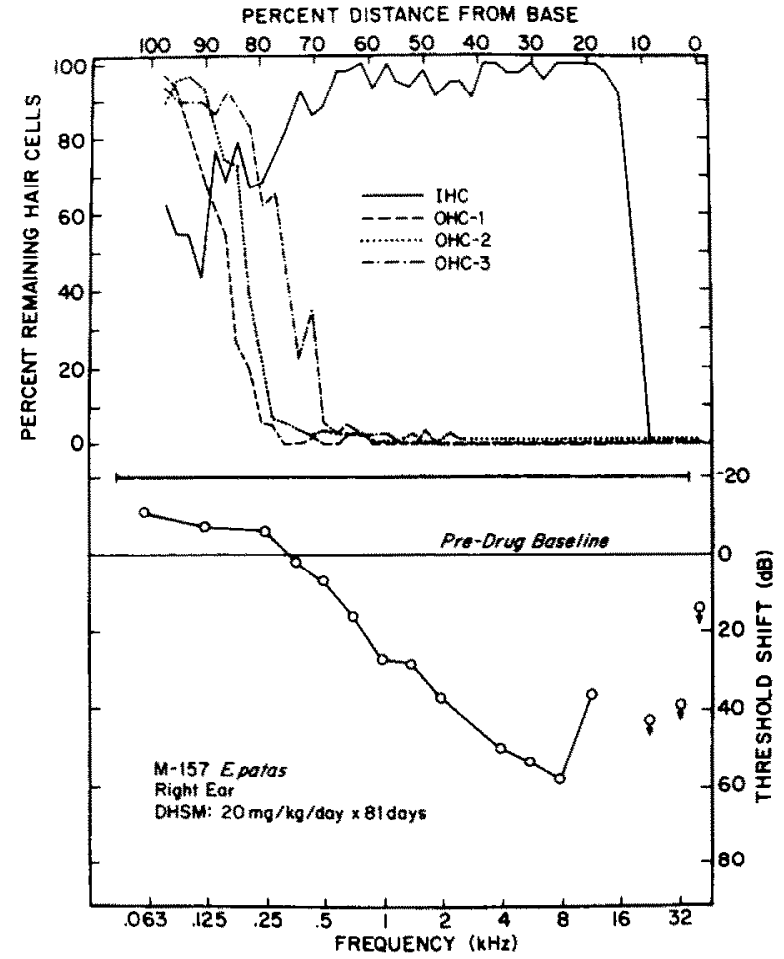

Fig. 5. Cytocochleogram for M-157 (upper panel) and corresponding final steady tone audiogram (lower panel). Actual length of basilar membrane, $22.17 \mathrm{~mm}$. sensitivity was present in the low-frequency tail. At day 40 , the tip was elevated by over $40 \mathrm{~dB}$ and yet little effect was seen at low frequencies. By day 41 , with a $50-\mathrm{dB}$ decrease in sensitivity at $\mathrm{CF}$, the highest frequencies of $4.4 \mathrm{kHz}$ and above were not measurable and an elevation at low frequencies was present. SPLs of greater than $110 \mathrm{~dB}$ SPL were not measured, though extrapolation from the $4.2 \mathrm{kHz}$ masker at $84 \mathrm{~dB}$ SPL to these higher levels at $4.4 \mathrm{kHz}$ yields a low-pass filter function.

Again, comparisons of normal PTCs at 10, 30, and $60 \mathrm{~dB}$ SL (right panel) and post-drug PTCs at roughly equivalent SPLs failed to explain these changes on the basis of increases in test signal levels. Even at the highest test level of $60 \mathrm{~dB} \mathrm{SL}$ the normal PTCs exhibited sharp bandpass tuning. This characteristic disappeared quite rapidly with increases in threshold for pathological PTCs.

Fig. 5 presents the final steady-tone audiogram (lower panel) and corresponding cytocochleogram (upper panel) for a second subject, M-157. The final hair cell count indicated no receptors present in the basal $10 \%$ of the cochlea; nearly normal numbers of IHCs from 15 to $75 \%$ distance from 


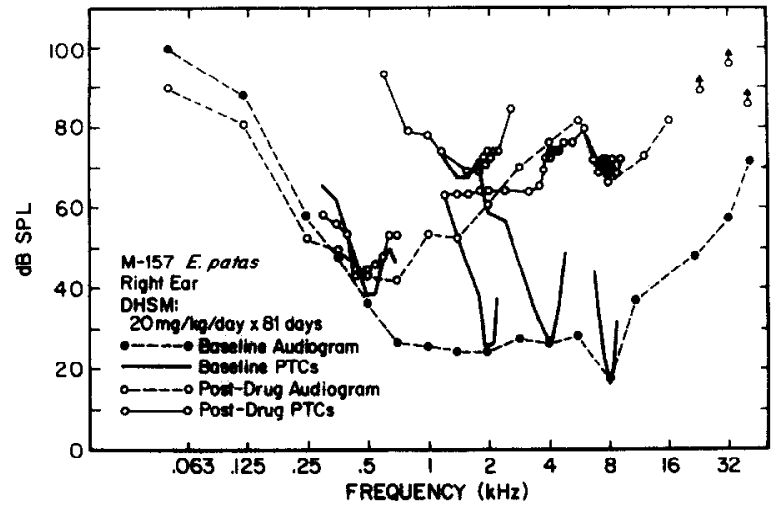

Fig. 6. Direct comparison of baseline and post-drug pulsed-tone threshold curves and PTCs for M-157.

the base, with no OHCs over the same areas. The condition of the IHCs in these regions appeared normal. There was a gradual increase in OHCs over the apical $20 \%$ of the organ of Corti. In this

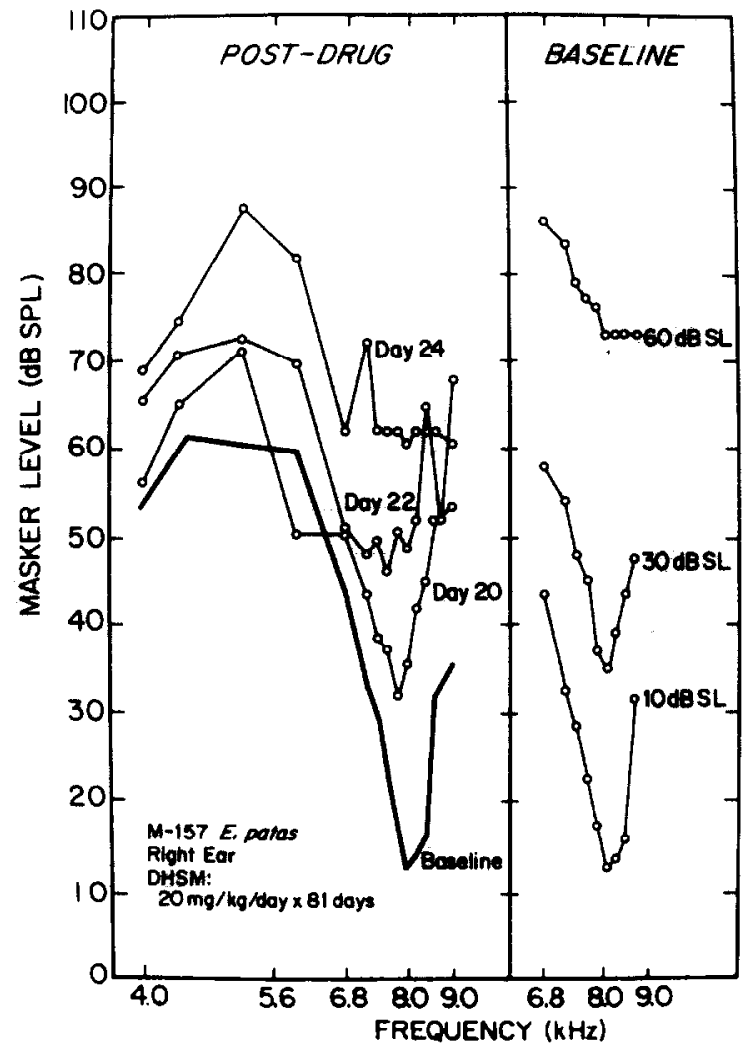

Fig. 7. Systematic changes in $8 \mathrm{kHz}$ PTC for $\mathbf{M}-157$ measured on various days post-drug (left panel). Normal PTCs measured for the same subject at 10,30 , and $60 \mathrm{~dB}$ SL are presented for comparison (right panel) from Smith et al. (1987).
OHC transition region, the number of IHCs dropped off to an approximately $50 \%$ loss at the apex. This reversal of relative population densities is common in the patas/DHSM model and was also seen in the cytocochleogram from another subject in this study that is not presented here.

The accompanying audiogram showed an increase in thresholds at $710 \mathrm{~Hz}$ and above, corresponding closely to regions of receptor damage. The maximum shifts in threshold were $40-60 \mathrm{~dB}$ at 2 to $11.2 \mathrm{kHz}$, from regions of complete $\mathrm{OHC}$ loss and IHC retention. The extent of the threshold shift prohibited measurement above $8 \mathrm{kHz}$. For frequencies of $250 \mathrm{~Hz}$ and below, a decrease in thresholds of 5-10 dB was evident. While evidence of this 'hyper-recovery' at frequencies adjacent to sites of extensive receptor losses is common, no explanation for this phenomenon is readily apparent. The slope of the threshold shift for M-157 is less steep than that for M-155 (see Fig. 1). The slope has proven to be related to the extent of the lesion: the further the hair cell loss progresses from the base, the greater the decrease in the slope of the threshold shift. The change in slope is most likely related to the relative decrease in slope of the excitation pattern on the basilar membrane with decreases in frequency.

The pre- and post-drug pulsed-stimulus threshold curves and PTCs for M-157 are compared in Fig. 6. The post-drug PTCs at 8 and 4 $\mathrm{kHz}$, with threshold elevations of over $50 \mathrm{~dB}$, followed the contour of the summary threshold curve, showing a complete lack of specificity. At 2 $\mathbf{k H z}$ the curve was greatly elevated and broadened, yet some evidence of tuning remained. As with M-155, this residual tuning may have been a function of overlap with the less damaged receptor populations where some tuning remains. The lowest frequency of masker in the post-drug $2-\mathrm{kHz}$ PTC tail was $600 \mathrm{~Hz}$, where thresholds were relatively unaffected by the drug. The PTC at $500 \mathrm{~Hz}$ showed an approximately 8-dB decrease in tip and high-frequency tail sensitivity and an increase in sensitivity of the low-frequency tail.

The progression of changes in the $8 \mathrm{kHz}$ PTC for M-157 is given in Fig. 7. As with the changes at other frequencies, there was an inordinate increase in the tip relative to low-frequency tail. Day 20 measurements indicated a $20-\mathrm{dB}$ elevation in 


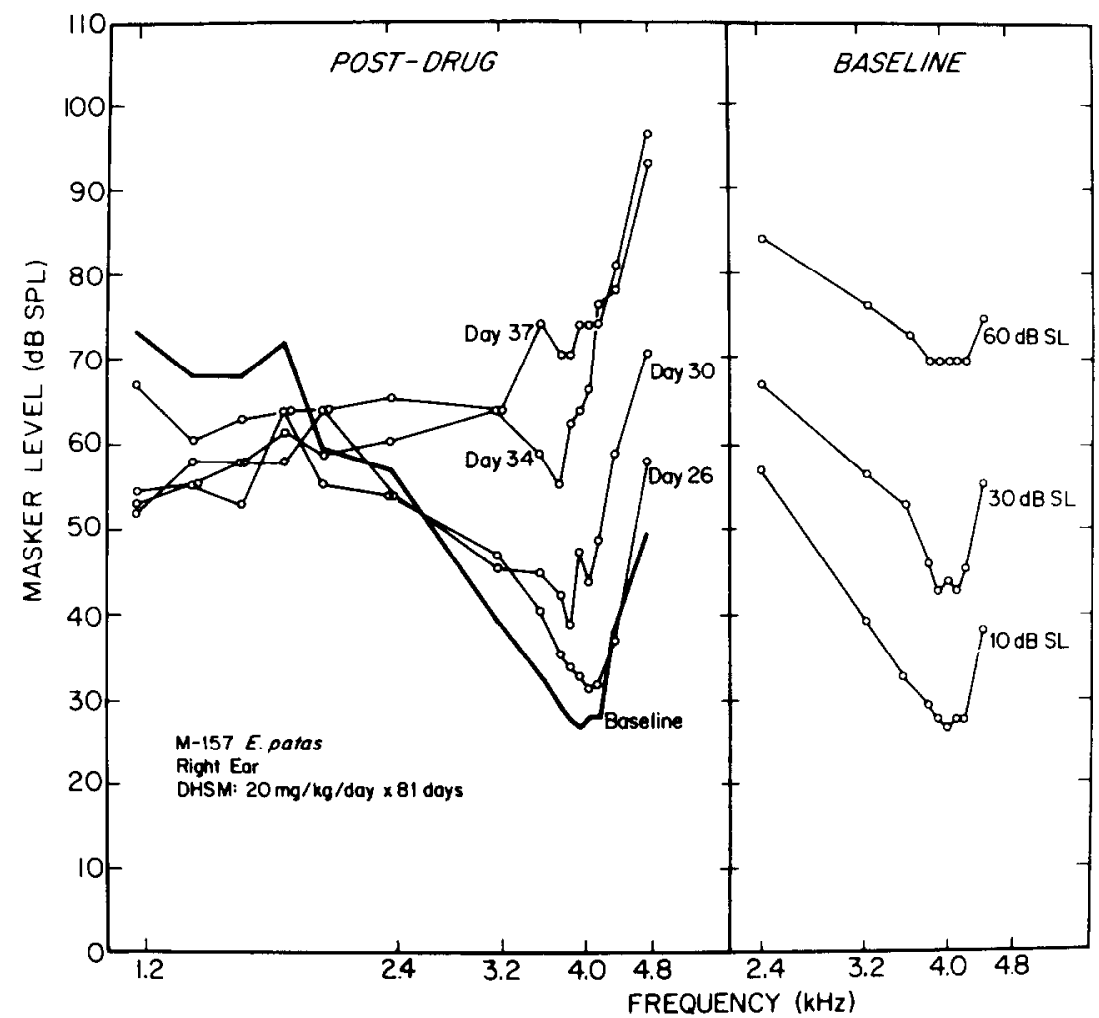

Fig. 8. Systematic changes in $4 \mathrm{kHz}$ PTC for M-157 measured on various days post-drug (left panel). Normal PTCs for same subject at 10, 30, and $60 \mathrm{~dB}$ SL are presented for comparison (right panel) from Smith et al. (1987).

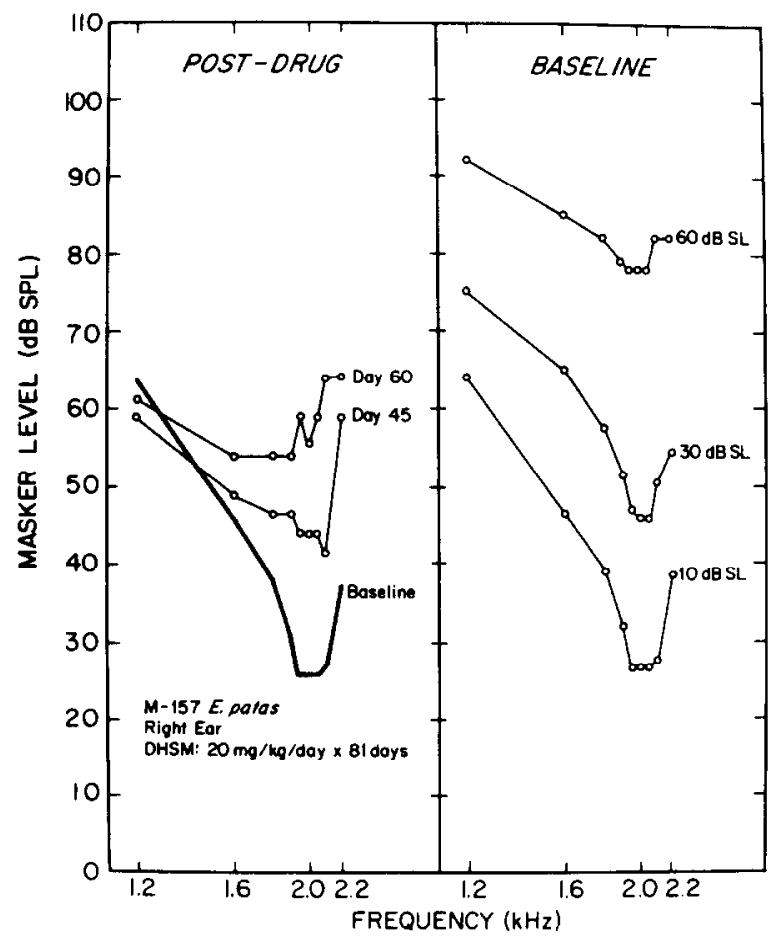

threshold, with a variable, yet probably insignificant, increase in the low-frequency tail. At days 22 and 24 an increase was present at both the tail and tip. The tip region was flat, and it was not possible to measure a high-frequency tail.

Unlike other frequencies for this and other subjects, a comparison of the pathological PTCs with baseline PTCs revealed some similarities, although the abnormal PTCs were somewhat broader. A direct comparison of the day 24 PTC with the pre-drug PTC at $60 \mathrm{~dB}$ SL showed a marked similarity, which, as seen in Fig. 6, may have been a result of the contour of the threshold curve.

The systematic changes seen at $4 \mathrm{kHz}$ for $\mathrm{M}-157$ were similar to those for the other animals. The individual data at $4 \mathrm{kHz}$ for this animal are shown

Fig. 9. Systematic changes in $2 \mathrm{kHz}$ PTC for M-157 measured on various days post-drug (left panel). Normal PTCs measured at 10,30 , and $60 \mathrm{~dB}$ SL for same subject are presented for comparison (right panel), from Smith et al. (1987). 
in Fig. 8. Decreases in tip sensitivity for all days were associated with a hypersensitivity of the low-frequency tail. For days 34 and 37, which showed increases at $4 \mathrm{kHz}$ of 40 and $48 \mathrm{~dB}$, respectively, the curves resembled low-pass filter functions.

Comparisons of the post-drug PTCs with those at equivalent SPLs indicate that pathological changes in tuning were quite unlike those associated with changes in signal level. Even at the highest level of $60 \mathrm{~dB}$ SL the normal tuning curve function exhibited relatively normal bandpass characteristics. Pathological PTCs lose this normal function rapidly with elevations of threshold.

At $2 \mathrm{kHz}$, for $\mathrm{M}-157$, the increase in threshold was gradual, requiring several weeks to produce a tip elevation of $30 \mathrm{~dB}$. Fig. 9 shows that at both days 45 and 60 , where the losses of sensitivity were 17 and $30 \mathrm{~dB}$ respectively, the tuning curves were low-pass.

A comparison of normal and pathological PTCs indicated that changes evident with increases in threshold were easily differentiated from those seen with increases in SPLs for the normal ear.

\section{Discussion}

The effects of selective OHC loss on psychophysical tuning curves can be summarized as follows: the initial effects were reliably seen as elevations of the PTC tip accompanied by a slight shift of $\mathrm{CF}$ towards lower frequencies. This selective tip elevation could be as much as a $40-\mathrm{dB}$ loss of sensitivity independent of changes in the low frequency tail which appeared normal or, frequently, hypersensitive. The low frequency tail also decreased in sensitivity with increases in thresholds of greater than $40-50 \mathrm{~dB}$. The slope of the high-frequency tail showed relatively little change although it was elevated in a linear fashion with an increase in threshold at CF. Following an increase of $50 \mathrm{~dB}$ or more at CF, the PTC exhibited a low-pass filter function.

Comparison of changes in PTCs due to increased SPLs with those associated with $O H C$ damage

Measures of frequency selectivity have been shown to be sensitive to changes in the absolute signal level at which measurements are taken ( Nel- son, 1980; Green et al., 1981; Nelson and Freyman, 1984). The shape of the PTC broadens and decreases in tip-to-tail depth as the absolute signal level is increased. Unlike changes brought about by pathology, significant tuning was observed and normal PTCs retain bandpass function even at the highest levels of $60 \mathrm{~dB}$ SL. Similar nonlinear behavior, for example, has been observed in IHC isoamplitude curves when the amplitude criterion is varied (Russell and Sellick, 1978). The basis of the level dependence of the hair cell, and subsequently of the system as a whole, is apparently the nonlinear mechanical action of the stereocilia (Khanna, 1984).

When comparisons are made of normal PTCs taken at high signal levels with pathological PTCs taken at roughly equivalent SPLs, it becomes obvious that the changes in PTCs following OHC loss cannot be accounted for on the basis of increased measurement levels. Following drug treatment, tuning curves undergo a rapid increase in threshold and nearly complete detuning in response to $\mathrm{OHC}$ loss. In the present study, this transition from low-threshold, sharply tuned PTC to highthreshold, low-pass filter can only be explained as a change in the tuning mechanism brought on by loss of $\mathrm{OHC}$ function.

Changes in PTC tip response associated with loss of outer hair cells

In these studies increases in threshold were invariably followed by a rapid and nearly complete loss of psychophysical tuning. The rate of increase in threshold varied across frequency and length of time post-drug, but could be as rapid as $10-20 \mathrm{~dB} /$ day. The pattern of change in tuning was systematic and entirely dependent upon threshold and was presumably a function of orderly alterations in the processing mechanisms.

With increases in threshold of up to $30-40 \mathrm{~dB}$, there was a selective elevation and broadening of the tip region in the PTC response. The CF was also shifted toward lower frequencies. The slope of the high-frequency tail remained relatively constant yet was elevated in a more-or-less linear fashion with increases in threshold at CF. Following threshold shifts of greater than $50 \mathrm{~dB}$, the tip response was completely absent and the filter took on low-pass characteristics. As can be seen from 
comparisons of the cytocochleograms and corresponding final audiograms, threshold shifts to both steady and pulsed tones were of the order of $40-60 \mathrm{~dB}$ at frequencies corresponding to regions completely lacking OHCs. This suggests that the final transition of the PTC to low-pass filter function concomitant with shifts in threshold of greater than $40-50 \mathrm{~dB}$ is a result of complete removal of $\mathrm{OHC}$ influence. Because of the orderly covariation of threshold and tuning, it is likely that these two processes are dependent on the same mechanisms, namely degeneration of OHCs. Following complete loss of OHCs the PTC exhibits the characteristics of a low-pass filter process.

The results of these studies agree with the data from previous physiological studies indicating the dependence of the sensitivity and sharply tuned response of the FTC on normal OHC function. Temporary or permanent inhibition or alteration of $\mathrm{OHC}$ activity results in a filter lacking a sharply tuned tip. Interference with normal cell metabolism by either asphyxia or hypoxia (Evans, 1974; Brown et al., 1983b), hypothermia (Brown et al., $1983 \mathrm{c}$ ), or infusion of poisons into perilymph (Evans and Klinke, 1974) produce a selective attenuation in the tip region of both IHC and VIIIth nerve fiber FTCs. In the extreme, these anomalous filter functions resemble low-pass filters. As has been shown by Evans (1975), these pathological VIIIth nerve responses show the same selectivity characteristics as basilar membrane transfer functions from traumatized cochleae, being broadly tuned and low pass (Békésy, 1960; Johnstone and Boyle, 1967; Rhode, 1971, 1973). It is likely that this residual low-pass tuning seen following complete degeneration of OHCs in both the behavioral and physiological responses reflects the passive mechanical interactions of the basilar membrane and surrounding fluids. Because of the remarkable similarity of FTCs and the PTCs in both normal and pathological ears, it is suggested that the processes that determine the tuning of the auditory system as a whole are primarily peripheral phenomena and very likely can be explained in terms of normal OHC function.

These data are also in agreement with those from human psychophysical analyses of changes in auditory frequency selectivity with cochlear pathology (c.f., Pick et al., 1977; Wightman, 1982;
Zwicker and Schorn, 1978; Zwicker, 1983). The human data have consistently shown a relationship between increases in threshold and loss of frequency selectivity similar to that shown with moderate losses of sensitivity in the present study. However, in this study the mechanisms for increases in threshold and loss of specificity are controlled and identified as the OHC subsystem. It is, however, difficult to reconcile these data with those of Dallos et al. (1977) which show no decrease in selectivity in areas of the basilar membrane with complete loss of OHCs.

While the intracellular data show that the $\mathrm{OHCs}$ are actually less sensitive than the IHCs (Dallos et al., 1982; Dallos, 1985), loss of OHC function results in a $40-60 \mathrm{~dB}$ loss of sensitivity in the system as a whole. While apparently paradoxical, these data are not at all inconsistent and lend support to the suggestion that $\mathrm{OHC}$ function is based on active cellular processes.

\section{PTC low-frequency tail response to selective lesion of $\mathrm{OHCs}$}

The data also indicate that moderate increases in threshold at CF produce a hypersensitivity in the response of the low-frequency tail of the PTC. This increase in sensitivity, which always accompanies a hyposensitivity of the tip response, is similar to that recently observed by Liberman and Dodds (1984) in single-unit FTCs arising from identified IHCs innervating areas of selective $\mathrm{OHC}$ damage or loss. Zwislocki (1984) suggests that a disruption in the normal shearing motion between the tectorial and basilar membranes, caused by depletion of OHCs, explains this finding. Since these same processes form the basis for psychophysical frequency selectivity, he suggests this same hypersensitivity should be obtainable in psychophysical tuning curves. These data, showing an increase in sensitivity of the low-frequency tail accompanying increases in threshold of $10-40 \mathrm{~dB}$, indicating initial selective insult to $\mathrm{OHCs}$, probably represent this same phenomenon.

\section{Summary}

These psychophysical data support the suggestions of Evans (1973, 1975), Evans and Wilson (1973, 1975), and Khanna (1984) that the 
observed physiological filter function is a composite of two interacting processes. The first being a low-pass filter process dependent upon the passive mechanical properties of the basilar membrane and dynamics of the surrounding fluids. This mechanical system is relatively immune to trauma. The second process, the so-called 'second filter,' is a sharply tuned response near the highfrequency cut-off of the first filter. This active process reflects the function of the OHC subsystem which is demonstrably physiologically labile.

The selectivity of the normal ear is determined by the mechanical influence of the sharply tuned OHC effectors on the low-pass, broadly tuned, basilar membrane motion. This frequency information is then communicated to the IHC receptors via disturbances in the endolymph. The response of the IHCs, and subsequently of the auditory afferents, directly reflects the influence of these processes. When the ear is subjected to temporary or permanent insult, the sharply tuned segment of the response is especially vulnerable and rapidly disappears (Brown et al., 1983a, b, c; Evans, 1970a, b, 1972; Evans and Klinke, 1974; Liberman and Dodds, 1984). What remains following removal of the influence of the second-filter process is the low-pass response of the basilar membrane. This residual tuning function is the same response reported in basilar membrane transfer functions from dead ears in living preparations or in human cadavers (Békésy, 1960; Johnstone and Boyle, 1967; Rhode, 1971, 1973), IHC responses during anoxia, hypothermia, and crossed olivocochlear bundle stimulation (Brown et al., 1983a, b, c), VIIIth nerve responses from areas of selective OHC loss or damage (Liberman and Dodds, 1984), and now, from the behavior of the system as a whole following $\mathrm{OHC}$ degeneration. This low-pass response is likely determined by the passive mechanical properties of the basilar membrane and dynamics of the surrounding fluids.

\section{Conclusions}

(1) The initial effects of damage to the OHC subsystem include a selective attenuation of the tuning curve tip together with an increase in the sensitivity of the low-frequency tail. The slope of the high-frequency tail remains relatively constant and is elevated as a linear process with elevations in threshold at CF.

(2) With increases in threshold of greater than $50 \mathrm{~dB}$, corresponding to a complete degeneration of OHCs, both the low-frequency tail and tip region are elevated and the resulting filter is lowpass.

(3) The residual low-pass function is likely a reflection of the mechanical tuning of the basilar membrane and dynamics of surrounding fluids and is the same as measured from IHCs, primary afferents, and basilar membrane in pathological preparations.

(4) The similarity between physiological and behavioral measures of frequency selectivity in normal ears and in response to controlled trauma suggest a common peripheral origin for tuning at the cellular level.

(5) The data suggest the IHC subsystem is responsible for reception of frequency specific information from the OHCs and control of the subsequent centripetal flow of that information.

\section{Acknowledgements}

The authors would like to express their gratitude to Drs. David J. Anderson, Josef M. Miller, William R. Uttal, and Joseph E. Hawkins, Jr., for their insightful review and comments during the course of these studies and analysis of the data. We would also like to thank Drs. Richard A. Altschuler and Lynn Carlisle and the Otopathology Laboratory at Kresge Hearing Research Institute for their skilled histological preparations. This research was supported by NIH grant no. NS05785 and a Horace H. Rackham Graduate School Dissertation Grant to D.W.S.

\section{References}

Békésy, G. von. (1960) Experiments in Hearing, McGraw-Hill, New York.

Brown, M.C., Nuttall, A.L. and Masta, R.I. (1983a) Intracellular recordings from cochlear inner hair cells: Effects of stimulation of the crossed olivocochlear efferents. Science 222, 69-72.

Brown, M.C., Nuttall, A.L., Masta, R.I. and Lawrence, M. (1983b) Cochlear inner hair cells: Effects of transient asphyxia on intracellular potentials. Hear. Res. 9, 131-144.

Brown, M.C., Smith, D.I. and Nuttall, A.L. (1983c) The temperature dependency of neural and hair cell responses 
evoked by high frequencies. J, Acoust. Soc. Am. 73, $1662-1670$.

Brownell, W.E. (1983) Observations on a motile response in isolated outer hair cells. In: Neural Mechanisms of Hearing, pp. 5-10. Editors: W.R. Webster and L.M. Aitkin. Monash University Press, Clayton, Australia.

Dallos, P. (1985) Response characteristics of mammalian cochlear hair cells. J. Neurosci. 5, 1591-1608.

Dallos, P., Ryan, A., Harris, D., McGee, T. and Özdamar, Ö. (1977) Cochlear frequency selectivity in the presence of hair cell damage. In: Psychophysics and Physiology of Hearing, pp. 249-258. Editors: E.F. Evans and J,P. Wilson, Academic Press, New York.

Dallos, P., Santos-Sacchi, J. and Flock, A. (1982) Intracellular recordings from cochlear outer hair cells. Science 218 , 582-584.

Evans, E.F. (1970a) Narrow 'tuning' of cochlear nerve fibre responses in the guinea pig. J. Physiol. 206, 14-15.

Evans, E.F. (1970b) Narrow 'tuning' of the responses of cochlear nerve fibres emanating from the exposed basilar membrane. J. Physiol. 208, 75-76.

Evans, E.F. (1972) The frequency response and other properties of single fibres in the guinea pig cochlea. J. Physiol. $226,263-287$.

Evans, E.F. (1973) The frequency selectivity of the cochlea. In: Basic Mechanisms in Hearing, pp. 519-551. Editor: A.R. Møller. Academic Press, New York.

Evans, E.F. (1974) The effects of hypoxia on the tuning of single cochlear nerve fibres. J. Physiol. 238, 65-67.

Evans, E.F. (1975) The sharpening of cochlear frequency selectivity in the normal and abnormal cochlea. Audiology 14 , $197-201$

Evans, E.F. and Klinke, R. (1974) Reversible effects of cyanide and furosemide on the tuning of single cochlear nerve fibres. J. Physiol. 242, 129-130.

Evans, E.F. and Wilson, J.P. (1973) The frequency selectivity of the cochlea. In: Basic Mechanisms of Hearing, pp. 519-554. Editor: A.R. Møller. Academic Press, New York.

Evans, E.F. and Wilson, J.P. (1975) Cochlear tuning properties: Concurrent basilar membrane and single nerve fiber measurements. Science 190, 1219-1221.

Flock, A. (1983) Review paper: Hair cells, receptors with a motor capacity? In: Hearing - Physiological Bases and Psychophysics, pp. 2-7. Editors: R. Klinke and R. Hartmann, Springer-Verlag, New York.

Green, D.M., Shelton, B.R., Picardi, M.C. and Hafter, E.R. (1981) Psychophysical tuning curves independent of signal level. J. Acoust. Soc. Am. 69, 1758-1762.

Johnstone, B.M. and Boyle, A.J.F. (1967) Basilar membrane vibration examined with the Mössbauer technique. Science $158,389-390$.

Khanna, S.M. (1984) Inner ear function based on the mechanical tuning of the hair cells. In: Hearing Science: Recent Advances, pp. 213-239. Editor: C.I. Berlin. College-Hill Press, San Diego.

Khanna, S.M. and Leonard, D.G.B. (1986) Relationship between basilar membrane tuning and hair cell condition. Hear. Res. 23, 55-70.
Leonard, D.G.B. and Khanna, S.M. (1984) Histological evaluation of damage in cat cochleas used for measurement of basilar membrane mechanics. J. Acoust. Soc. Am. 75. $515-527$

Liberman, M.C. and Dodds, L.W. (1984) Single-neuron labeling and chronic cochlear pathology. III. Stereocilia damage and alterations of threshold tuning curves. Hear. Res. 16. 55-74.

Moody, D.B., Beecher, M.D. and Stebbins, W.C. (1975) Behavioral methods in auditory research. In: Handbook of Auditory and Vestibular Research Methods, pp. 439-495. Editors: J.A. Vernon and C.A. Smith. Charles Thomas, Springfield, II.

Nelson, D.A. (1980) Comment on: The use of psychophysical tuning curves to measure frequency selectivity. In: Psychophysical, Physiological, and Behavioral Studies in Hearing, pp. 116-117. Editors: G. van den Brink and F.A. Bilsen. Delft University Press, Delft.

Nelson, D.A. and Freyman, R.L. (1984) Broadened forward-masked tuning curves from intense masking tones: Delay-time and probe-level manipulations. J. Acoust. Soc. Am. 75, 1570-1577.

Pick, G., Evans, E.F, and Wilson, J.P. (1977) Frequency resolution in patients with hearing loss of cochlear origin. In: Psychophysics and Physiology of Hearing, pp. 273-282. Editors: E.F. Evans and J.P. Wilson. Academic Press, London.

Rhode, W.S. (1971) Observations of the vibration of the basilar membrane in squirrel monkeys using the Mössbauer technique. J. Acoust. Soc. Am. 49, 1218-1231.

Rhode, W.S. (1973) An investigation of post-mortem cochlear mechanics using the Mössbauer effect. In: Basic Mechanisms in Hearing, pp. 49-63. Editor: A.R. Maller. Academic Press, New York.

Russell, I.J. and Sellick, P.M. (1978) Intracellular studies of hair cells in the mammalian cochlea. J. Physiol. 284, $261-290$.

Serafin, S.V., Moody, D.B. and Stebbins, W.C. (1982) Frequency selectivity of the monkey's auditory system: Psychophysical tuning curves. J. Acoust. Soc. Am. 71, 1513-1518.

Smith, D.W., Moody, D.B. and Stebbins, W.C. (1987) The effects of changes in absolute measurement level on psychophysical tuning curves in quiet and noise in patas monkeys. J. Acoust. Soc. Am. (in press).

Wightman, F.L. (1982) Psychoacoustic correlates of hearing loss. In: New Perspectives on Noise-Induced Hearing Loss, pp. 375-393. Editors: R.P. Hamernik, D. Henderson, and R. Salvi. Raven Press, New York.

Wightman, F.L., McGee, T. and Kramer, M. (1977) Factors influencing frequency selectivity in normal and hearing impaired listeners. In: Psychophysics and Physiology of Hearing, pp. 295-306. Editors: E.F. Evans and J.P. Wilson. Academic Press, New York.

Zenner, H.P., Zimmerman, U. and Schmitt, U. (1985) Reversible contraction of isolated mammalian cochlear hair cells. Hear. Res. 18, 127-133. 
Zwicker, E. (1974) On a psychophysical equivalent of tuning curves. In: Facts and Models in Hearing, pp. 132-140. Editors: E. Zwicker and E. Terhardt. Springer-Verlag, New York.

Zwicker, E. (1983) On peripheral processing in human hearing. In: Hearing - Physiological Bases and Psychophysics, pp.
104-109. Editors: R. Klinke and R. Hartmann. SpringerVerlag, New York.

Zwicker, E. and Schorn, K. (1978) Psychoacoustic tuning curves in audiology. Audiology 17, 120-140.

Zwislocki, J.J. (1984) How OHC lesions can lead to neural cochlear hypersensitivity. Acta Oto-Laryngol. 97, 529-534. 